\title{
Empirical evidence for key hosts in persistence of a tick-borne disease
}

\author{
Sarah E. Perkins ${ }^{\mathrm{a}, *}$, Isabella M. Cattadori ${ }^{\mathrm{a}}$, Valentina Tagliapietra ${ }^{\mathrm{b}}$, \\ Annapaola P. Rizzoli ${ }^{\mathrm{b}}$, Peter J. Hudson ${ }^{\mathrm{a}}$ \\ ${ }^{\mathrm{a}}$ Institute of Biological Sciences, University of Stirling, Stirling, Scotland FK9 4LA, UK \\ ${ }^{\mathrm{b}}$ Centro di Ecologia Alpina, 38040 Viote del Monte Bondone, Trento, Italy
}

Received 14 February 2003; received in revised form 5 May 2003; accepted 7 May 2003

\begin{abstract}
An important epidemiological consequence of aggregated host-parasite associations occurs when parasites are vectors of pathogens. Those hosts that attract many vectors will tend to be the focus of transmission. But to what extent, and can we identify characteristics of these key hosts? We investigated these questions with respect to the host-tick relationship of the yellow-necked mouse, Apodemus flavicollis, a critical host in the maintenance of the zoonotic disease, tick-borne encephalitis. Transmission of the virus occurs when ticks feed in a 'co-feeding' aggregation. Thus, the number and frequency of co-feeding groups provides an estimate of the potential rate of virus transmission. We recorded the spatio-temporal variations in co-feeding on a population of rodents in conjunction with recording individual host characteristics. Using Lorenz curves, we revealed conformation of tick-borne encephalitis transmission potential to the 20/80 Rule, where the $20 \%$ of hosts most infested with ticks were accountable for $80 \%$ of transmission potential. Hosts in the transmission cohort were identified as the sexually mature males of high body mass. Therefore control efforts targeted at this group would substantially reduce transmission potential compared to non-targeted control of the population, which resulted in a linear reduction in transmission potential. Focusing on the 'wrong' functional group would have little impact upon transmission potential until a considerable proportion of the population had been subject to control. However, individuals can change their functional status over time making it difficult to predict the contribution of these individuals to future transmission.
\end{abstract}

(C) 2003 Australian Society for Parasitology Inc. Published by Elsevier Science Ltd. All rights reserved.

Keywords: Heterogeneity; Vector-borne disease; 20/80 Rule; Co-feeding; Tick-borne encephalitis

\section{Introduction}

The key to understanding and controlling the spread of lethal pathogens is to identify how the disease flows through the host population and more specifically which individuals are responsible for the majority of the transmission. If we can identify these key individuals then we could target disease control efforts. In the case of vector-borne disease transmission the key hosts are expected to be those that support large numbers of vectors. A particularly important heterogeneity in macroparasite-host systems is the observation that the majority of hosts harbour a minority of the parasites and the minority of hosts support the majority of the macroparasite population (Shaw and Dobson, 1995). The propensity of parasites to aggregate within their host population is borne out of the heterogeneities within the

\footnotetext{
* Corresponding author. Tel.: +44-1786-473171x7813; fax: +44-1786464994.

E-mail address: s.e.perkins@stir.ac.uk (S.E. Perkins).
}

biological system and is a balance between endogenous features associated with host susceptibility and exogenous forces associated with variations in exposure to infection (Wilson et al., 2002). The resulting distribution of this hostparasite association can typically be described and modelled using a negative binomial distribution (Shaw and Dobson, 1995; Shaw et al., 1998).

Woolhouse et al. (1997) examined contact rates between vectors and their hosts and showed that not only were these highly variable between individual hosts but also conformed to the negative binomial distribution. This had a major impact on the basic reproductive number, $R_{0}$, an epidemiological parameter that provides us with a quantitative assessment of the ability of a pathogen to invade or persist within a population. Where $R_{0}>1$ an infection can establish and persist in a population whereas if $R_{0}<1$ an infection cannot invade or establish in a population.

From ten data sets of pathogens with frequencydependent transmission, Woolhouse et al. (1997) identified that $20 \%$ of hosts contributed $80 \%$ of the transmission 
potential, conforming to the '20/80 Rule'; a statistical pattern associated with a range of phenomena (e.g. Koch, 2000). This pattern has important consequences for the control of parasites and pathogens, since if we can identify and target the $20 \%$ of hosts that account for disease transmission, we may have a major influence on $80 \%$ of disease transmission, to the extent that $R_{0}$ falls below unity. As such, a pressing question in vector-borne disease transmission is to ask "Who are the hosts that contribute most to disease transmission?"

Numerous empirical studies have examined exposure and susceptibility of mammalian hosts to parasites and parasitic infection. These lead us to expect that sexually mature males, that consequently are old and of large body mass, are the functional group most likely to be heavily parasitised (Arneberg et al., 1998; Moore and Wilson, 2002; Wilson et al., 2002). As such these hosts are likely to constitute the vital few in terms of vector-borne transmission potential, whilst the small young females of the population are expected to constitute the trivial many and play little role in the transmission potential. Thus, in this study we classify functional groups in terms of a host's sex and sexual status.

Our hypothesis was that we could quantify the proportion of hosts contributing to transmission in terms of the '20/80 Rule'. Furthermore this transmission cohort would consist predominantly of the functional group that included large, sexually mature males. The mutually exclusive, alternative hypothesis is that spatial variability in ticks would explain a large proportion of the total aggregation such that the role of the individual would be relatively small (Haukisalmi and Henttonen, 1999). To test between these hypotheses we apportioned temporal and spatial aggregation according to the relative contribution that an individual imposed on the distribution to the overall variance using a generalised linear mixed model (GLMM) (Boulinier et al., 1996; Haukisalmi and Henttonen, 1999; Elston et al., 2001).

We examined transmission patterns through a capturemark-recapture (CMR) study of the yellow-necked mouse, Apodemus flavicollis, and their associated infestations with the sheep tick, Ixodes ricinus, the vector of the zoonotic disease tick-borne encephalitis (TBE) (Labuda et al., 1997). This disease is second only to Lyme disease as the most important vector-borne zoonotic disease in Europe with 10,000 human cases per annum reported from mainland Europe (http://www.tbe-info.com). TBE provides a good model system for testing our hypotheses since we can use non-invasive means of identifying potential TBE transmission. TBE virus is transmitted to susceptible ticks when they feed adjacent to infected ticks, through a process known as co-feeding which does not involve virus amplification within the host (Jones et al., 1987; Labuda et al., 1993). Transmission is facilitated by tick saliva and occurs only on specific hosts, for example, A. flavicollis (Labuda et al., 1997) and depends on cellular events such as invasion of immune cells that are transported to the lymph nodes. In the absence of co-feeding and suitable nonviraemic hosts, the threshold for disease persistence (measured by the reproductive number, $R_{0}$ ), falls below unity and TBE fades out (Randolph et al., 1996).

In our study we asked three questions: "What proportion of the host population is potentially involved with TBE transmission?" "Can we identify the functional group associated with the greatest potential for transmission?" and if so, "Do individual hosts remain in the transmission cohort?"

\section{Methods}

\subsection{Study area}

Our study was carried out at the Southern edge of the TBE virus distribution, in an isolated TBE focus (Hudson et al., 2001). The area is located in Valle dei Laghi, Trentino (grid reference, 1652050E 5093750N) in the north-eastern Italian Alps. The area was an isolated calcareous ridge (750-800 $\mathrm{m}$ a.s.l) that consisted predominantly of mixed broadleaf woodland dominated by mature beech Fagus sylvaticus, trees with sparse under-storey and very little ground flora.

\subsection{Rodent monitoring}

Intensive live trapping of woodland rodents was undertaken for two consecutive nights, fortnightly between 5 April and 18 October 2000 and an additional trapping session in the second week of November. Trapping effort consisted of 4,960 trap nights and resulted in 790 captures of 268 individual rodents. A total of five randomly selected sample areas $(8 \times 8$ trapping grids, with a $15 \mathrm{~m}$ inter-trap interval, i.e. 64 traps per grid) were used to monitor the rodent population. The trapping grids were located in similar habitats, and separated by a distance of at least $300 \mathrm{~m}$ with geographical features, such as a tracks or meadows, between them to reduce the likelihood of movement between grids and maintain independence of grids. All captures were made using Ugglan multiple capture traps (Special Mouse Trap 2, Grahnab, Sweden). Traps were baited with standardised pieces of potato and wild birdseed mixture (cereals and legumes); we did not pre-bait traps. Hay was provided for bedding and shelter.

Individual rodents were monitored using CMR techniques. On initial capture each individual was injected with an implantable subcutaneous passive induced transponder (PIT) tag (Trovan ID 100, Ghislandi and Ghislandi, Italy). For each capture a careful assessment of tick stage, abundance and distribution was carried out, recording location and composition of tick assemblages on each ear, body and the head. We defined co-feeding as an aggregation of any tick life stage feeding with one or more nymphs or adults with an imperceptible distance between them. Individual 
host characteristics recorded included: species, sex, breeding condition, and body mass. We classified adult A. flavicollis as individuals in breeding condition, i.e. descended testes for males and perforated vagina or pregnant for females.

\subsection{Analyses}

The transmission potential of parasites is usually quantified as the basic reproduction number, $R_{0}$ (Anderson and May, 1991). In terms of TBE infection dynamics $R_{0}$ represents the number of infected nymphs produced by a single infectious nymph in a fully susceptible tick population. We approximated $R_{0}$ and hence transmission potential using Eq. (1), as defined by Woolhouse et al. (1997)

$R_{0} \propto \sum_{i=1}^{m} \frac{v_{i}^{2}}{h_{i}}$

where ticks bite individual hosts $i$ and $i=1$ to $m$, where $m$ is the number of hosts. $v_{i}$ is the proportion of ticks sampled on host $i$ and $h_{i}$ is the proportion of hosts.

The empirical data were rank ordered from most to least tick-infested and $R_{0}$ was calculated for each host. Individuals were sequentially removed from the distribution and the corresponding cumulative reduction in transmission potential was calculated and plotted using Lorenz curves. This is essentially a graphical method of representing any form of inequality within a population (See Poulin (1993) for detailed methods of an application to hostmacroparasite distributions). This method of measuring inequality was first developed to quantify inequalities of income (Lorenz, 1905) and features heavily in the fields of economics and health. The extent of the inequality within a distribution as represented by a Lorenz curve can be quantified by a Gini coefficient (Lorenz, 1905; Poulin, 1993). In terms of our study the Gini coefficient captures deviation from equality, where zero represents perfect equality and unity implies all the transmission is focussed upon a single host. Parasitologists have utilised both Lorenz curves and Gini coefficients as methods for quantifying parasite aggregation (Poulin, 1993) and transmission inequality (Woolhouse et al., 1997). Here, we calculate inequality with respect to the transmission potential $\left(R_{0}\right)$ of the population.

Lorenz curves were calculated using firstly the entire tick burden of each host, and secondly using the ticks observed only in co-feeding groups. 'Removal' of hosts in this sense refers to exclusion of a host from the data set, which indicates it no longer supports parasites; a practical reality brought about by, for example, acaracide treatment of a rodent host.

To mimic a targeted control program we removed functional groups of hosts from the data set in order of greatest importance in terms of transmission potential, but randomly within a functional group. Conversely, we applied this method to sequentially removing functional groups in order of least importance in transmission potential. Finally, to mimic a control program that has no specific host targets the data set was randomised and transmission potential calculated.

TBE endemic cycles in A. flavicollis are maintained via the co-feeding process (Labuda et al., 1997). Therefore we investigated the characteristics of hosts with co-feeding, by use of a GLMM via an IRREML procedure (Genstat 4.2, 5th Edition) with binomial errors and co-feeding presence as the response variate. We investigated whether host sex, sexual status, body mass and larval tick burden of the host explained the variation seen in co-feeding presence. Population level explanatory variables included whether or not the host's tick intensity placed it in the tail of the negative binomial distribution (i.e. top $20 \%$ most larval infested hosts). These variables were entered as fixed effects into the model, as were their two-way interactions. Since preliminary analyses indicated that the majority of co-feeding ticks were concentrated in the tail of the hostlarval tick frequency distribution we repeated the GLMM procedure with the top $20 \%$ most larval infested hosts as the independent variable and explanatory variables as above as fixed effect components.

Assuming that a blood meal lasts 4-5 days (Sonenshine, 1992; Randolph, 1998), ticks observed on an individual in a subsequent trapping session (2 weeks later) were considered an independent tick count. However, these tick counts do constitute temporal and spatial pseudoureplication of individual hosts (Crawley, 2002). To overcome the possibility that autocorrelation in the multiple trapping data points could still cause problems of independence of the data each animal was fitted as a random effect in all GLMM models. This statistical technique allowed us to determine whether individual hosts were consistently observed within the tail of the distribution. Additionally, to account for spatiotemporal effects we determined the residual variation due to trapping week and trapping grid by entering these factors as random effects in all GLMM models.

\section{Results}

\subsection{What proportion of the host population is potentially involved with TBE transmission?}

The aggregated frequency distribution of larvae and co-feeding groups on captured rodents $(n=790)$ showed clear heterogeneities with the minority of hosts supporting the majority of co-feeding groups (Fig. 1). The distribution was not significantly different from a negative binomial distribution $(P=0.17)$, with a corrected moment estimate $(k)$ of 1.63. The heterogeneity in vector-host contact rates and the aggregation of co-feeding groups on heavily tickinfected hosts created non-linearities in transmission 


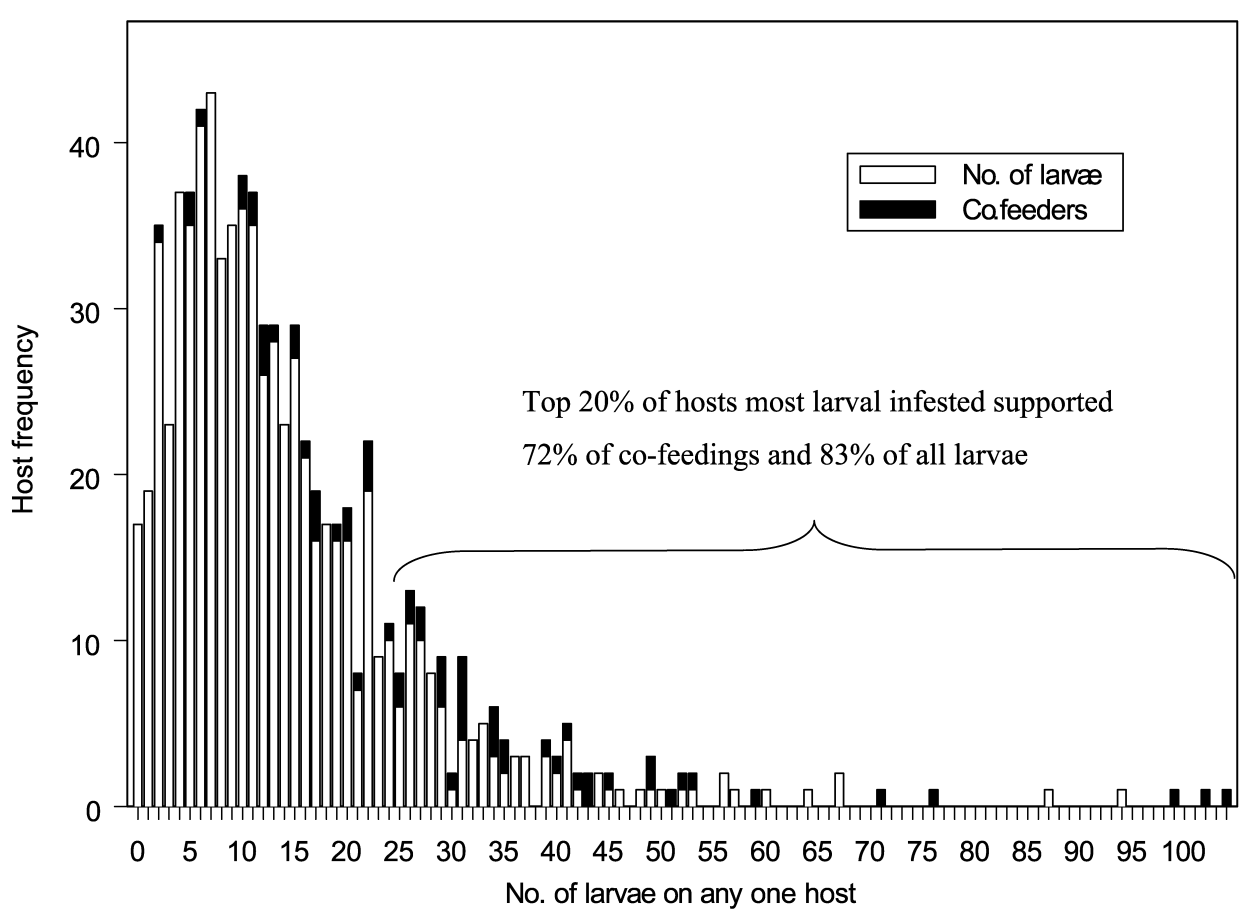

Fig. 1. Aggregated frequency distribution of ticks on A. flavicollis in a TBE focus (data presented as in Randolph et al., 1996). Hosts co-incidentally supporting co-feeding groups are shown in black. The non-linearities within the host-parasite frequency distribution were such that $72 \%$ of all co-feeding aggregations and $83 \%$ of all larvae were observed within the top $20 \%$ most larval infested hosts.

potential within the population (Fig. 2). Where all the ticks observed on rodent hosts were regarded as susceptible we found $20 \%$ of the population were responsible for $74 \%$ of transmission potential. However, when we considered ticks observed only in co-feeding groups, $20 \%$ of the population accounted for $94 \%$ of transmission (Fig. 2). These two estimates represent our upper and lower limits of the inequality of transmission in the host population and have a Gini coefficient of 0.53 and 0.78 , respectively. A Gini coefficient $>0.60$ indicates that a population conforms to the 20/80 Rule (Woolhouse et al., 1997). The transmission pattern of our population must exist somewhere between these two gradients, which accommodates the 20/80 Rule.

\subsection{Can we identify individuals or functional groups associated with the potential for transmission?}

With our first model we asked the question "which hosts supported co-feeding ticks?", and hence transmission potential. Of the random terms both host and temporal heterogeneity contributed significant variation to the incidence of co-feeding on hosts (Host: $\chi^{2}=2,609 ; 1$ d.f. $P<0.01$ Time: $\chi^{2}=1,239 ; 1$ d.f. $P<0.01$ respectively), but spatial variation was not significant $\left(\chi^{2}=2.4 ; 1\right.$ d.f. $P>0.05)$. Individuals are therefore important in determining variation in co-feeding, and hence transmission of TBE virus. The direction of the statistically significant ecological attributes of the hosts indicated that sexually mature males of high body mass and with high larval intensity were the functional group most likely to support co-feeding transmission (Table 1). Descriptively, larval intensity of hosts supporting co-feeding was higher than those not supporting co-feeding (geometric mean $\pm \mathrm{SE}$ of co-feeders and non-co-feeders, respectively; mean $=29.11 \pm 0.01$; mean $=10.20 \pm 0.17$ ).

As we have already quantified the majority of transmission potential in terms of the $20 \%$ of hosts most infested with larvae we investigated the characteristics of hosts in this section of the distribution. Firstly, we found that the random terms associated with spatio-temporal variability contributed significant variation to the occurrence of hosts in the 'top 20\%' (Trapping Grid: $\chi^{2}=18.2 ; 1$ d.f. $P<0.01$ Time: $\chi^{2}=163.2 ; 1$ d.f. $\left.P<0.01\right)$. Individual hosts

Table 1

Characteristics of hosts supporting co-feeding groups of ticks as determined by a generalised linear mixed model

\begin{tabular}{llll}
\hline Model term & $\begin{array}{l}\text { Wald statistic } \\
\left(\chi^{2}\right)\end{array}$ & d.f. & $P$ value \\
\hline Sex $*$ larval tick intensity & 30.75 & 1 & 0.001 \\
Sex $*$ breeding condition & 18.17 & 1 & 0.001 \\
Sex $*$ top 20\% & 33.83 & 1 & 0.001 \\
Body mass * larval intensity & 17.41 & 1 & 0.001 \\
Breeding condition $*$ larval tick intensity & 47.24 & 1 & 0.001 \\
Breeding condition $*$ body mass & 6.03 & 1 & 0.01
\end{tabular}

Response: co-feeding ticks. Model type: IRREML (GLMM), (Random terms $=$ week, grid, host) 


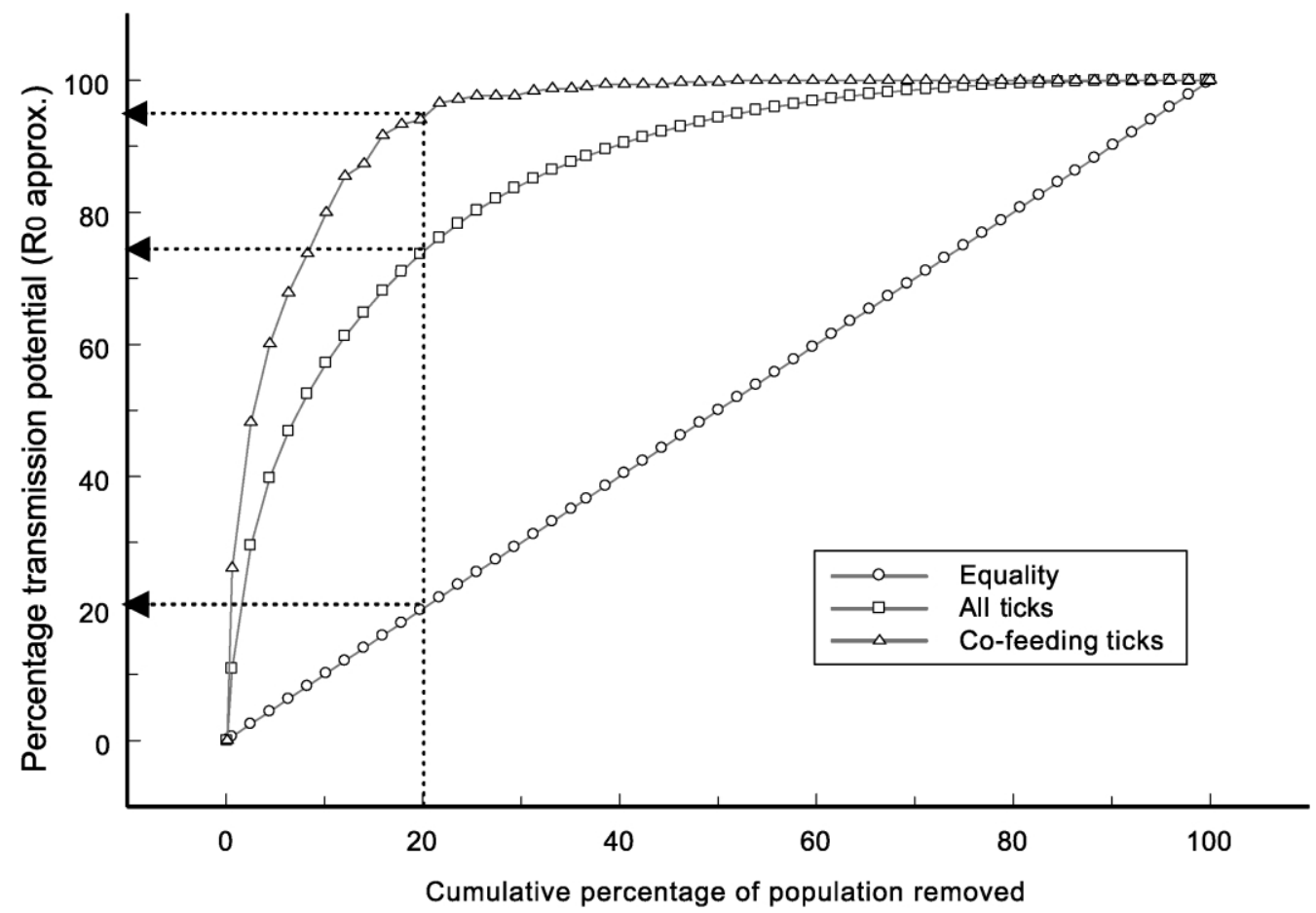

Fig. 2. Lorenz curves showing the reduction in transmission potential associated with sequential removal of hosts from the population. The degree of curvature indicates how the distributions differ from equality. For all ticks observed on hosts $20 \%$ of the population was accountable for $74 \%$ of transmission potential, Gini coefficient $=0.53$. For ticks observed only in co-feeding aggregations $20 \%$ of the population was responsible for $94 \%$ of transmission potential, Gini coefficient $=0.78$. A theoretical distribution is shown, representing a homogenous population, where the Gini coefficient is 0 .

explained a significant amount of deviance in the model and as such were found to remain in the transmission cohort, i.e. top $20 \%,\left(\chi^{2}=180.8 ; 1\right.$ d.f. $\left.P=0.001\right)$. Defining these hosts in terms of their functional groups we found a significant interaction between sex and sexual maturity. The group most accountable for disease transmission was found to be the sexually mature males $\left(\chi_{1,782}^{2}=7.60 ; P=0.01\right)$, (Fig. 3). For all functional groups the likelihood of being in the transmission cohort increased with increasing body mass, $\left(\chi_{1,782}^{2}=4.54 ; P=0.04\right)$. Other variables tested were found to be non-significant. Therefore sexually mature males of high body mass were the individuals that were contributing the majority of transmission potential.

By contrast, and as predicted from our hypothesis, sexually mature females were unlikely to be involved in transmission potential since they were the least likely of the functional groups to be in the tail of the distribution (Fig. 3). Surprisingly, when both sexes were sexually immature it was the females that were predicted to feature in transmission more than the males (Fig. 3). However, a clear sexbias in transmission potential was found with males having a mean co-feeding intensity twice as high as females (geometric mean $\pm \mathrm{SE}$ of males and females were $0.06 \pm 0.01$ and $0.03 \pm 0.01$, respectively).

Repeating the Lorenz curve procedure by removing functional groups of hosts in order of their importance in terms of transmission potential (Fig. 4) we effectively reduced transmission potential by $75 \%$ with removal of $20 \%$ of the population, $(\mathrm{Gini}=0.54)$ again approximating to the 20/80 Rule. When we randomised the distribution the reduction in transmission potential associated with $20 \%$ of the population was $29 \%$, (Gini $=0.03)$ i.e. the Lorenz curve was close to an equitable distribution (Fig. 4). Running a further simulation by sequentially removing the functional groups least involved in transmission potential, but randomly within these groups, we found the effective reduction in transmission potential associated with $20 \%$ of the population was just 7\% (Fig. 4).

\section{Discussion}

Within this disease system we identified distinct functional groups that were probably responsible for the majority of disease transmission in that neither tick bites nor co-feeding tick aggregations within the rodent population were random (Figs. 1 and 3). Our data indicated that the removal of the top $20 \%$ most tick-infested individuals should reduce the TBE transmission potential by $74-94 \%$ (Fig. 2). Since transmission does not occur on hosts without co-feeding ticks, the co-feeding data, and hence our uppermost estimate, should provide a good proxy for the potential transmission pattern. With a Gini coefficient of 0.78 , this pattern complied with the 20/80 Rule and was comparable 


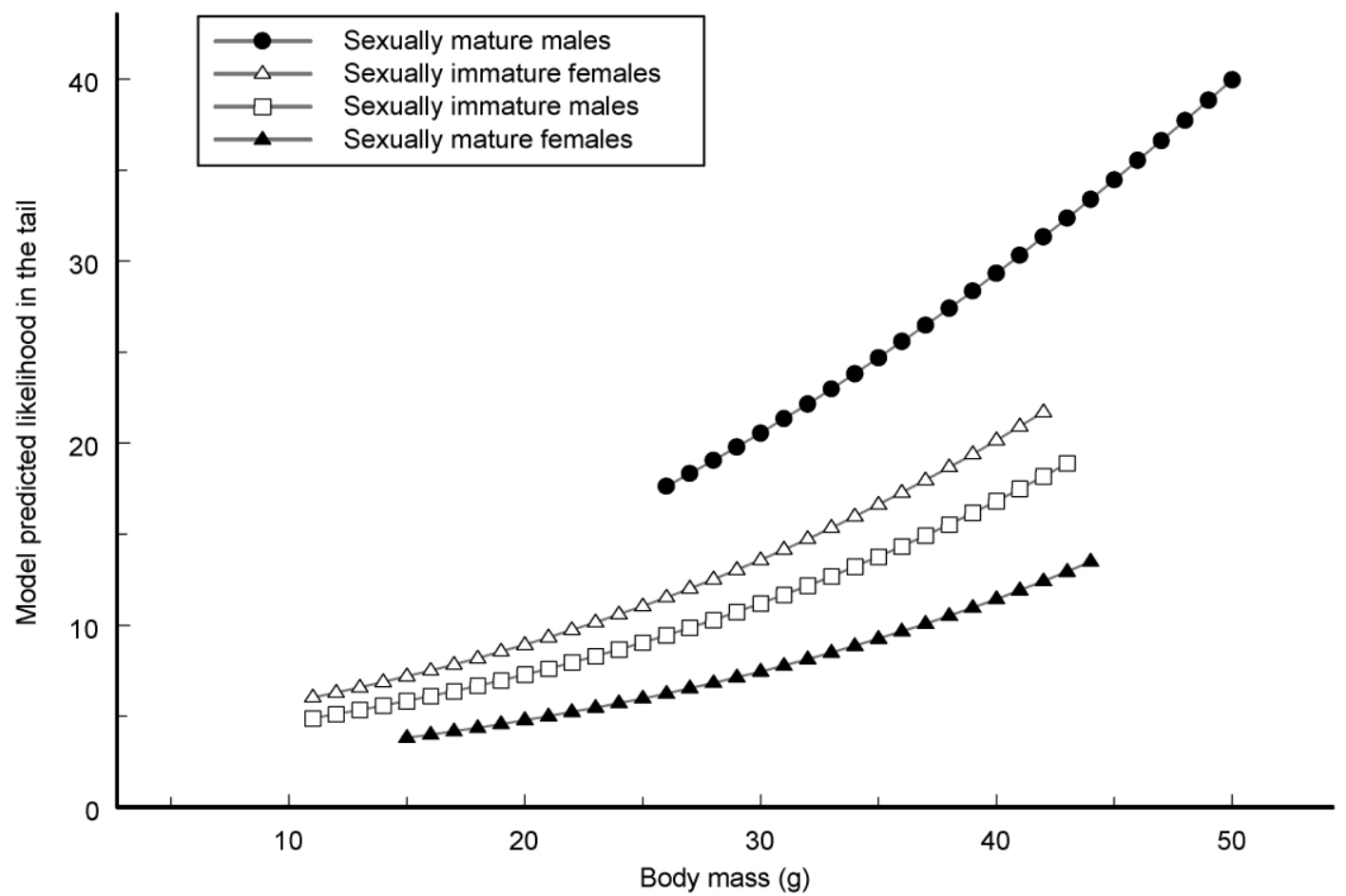

Fig. 3. Generalised linear mixed model predictions for males and females of differing breeding condition (at mean density/ha) and their likelihood of being in the tail of the distribution with increasing host body mass $(g)$. The body mass is displayed at the upper and lower limits expected for each functional group.

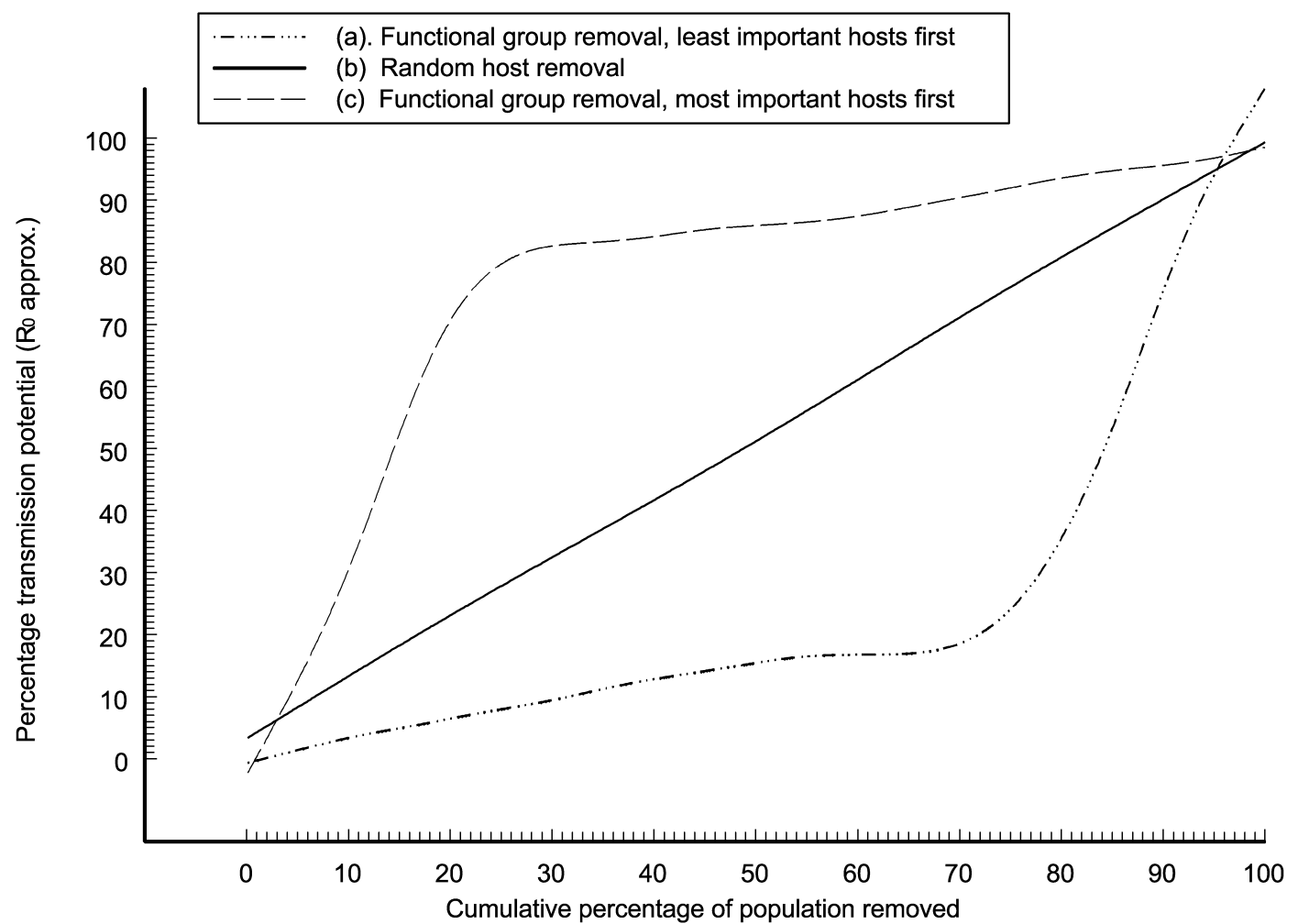

Fig. 4. Lorenz curves showing the reduction in transmission potential associated with sequential removal of hosts supporting co-feeding ticks from the population. The three distributions show differing patterns of transmission potential that could be generated from the same host-parasite distribution: (a) Targeted control of the 'wrong' host: $20 \%$ of hosts were accountable for $7 \%$ of transmission potential. (b) Random control: $20 \%$ of hosts were accountable for $29 \%$ of transmission potential. (c) Targeted control of the 'correct' hosts: $20 \%$ of hosts were accountable for $75 \%$ of transmission potential. 
with indices of other vector-borne diseases (Woolhouse et al., 1997) as well as patterns of co-feeding ticks on rodents in a comparable longitudinal study (Randolph et al., 1999). A Gini coefficient of 0.78 implies a high transmission inequality such that removal of just a small proportion of the population results in a substantial reduction in $R_{0}$. The importance of this finding for disease control has been emphasised by Woolhouse et al. (1997). However, they stressed the utility of this observation depends upon being able to identify the sub-groups in the population upon which to target our control efforts.

Our analyses qualitatively described the transmission cohort as consisting of sexually mature males of high body mass (Fig. 3). These individuals hosted high parasitic intensities whilst simultaneously supporting transmission of TBE via co-feeding ticks. Removal of the sexually mature males, (forming $26 \%$ of the total sample of individuals captured during the study), from the data set dramatically reduced transmission potential (and hence $R_{0}$ ) by $79 \%$. Such that the role the remaining population plays in terms of the co-feedings they support may be insignificant in TBE virus maintenance (Fig. 4). Randolph et al. (1999) calculated $R_{0}$ for TBE, and found it fell between one and 11 for a range of empirical rodent-tick distributions. Using the same calculation for our data we found $R_{0}$ of TBE to be 2.56. Reducing $R_{0}$ to unity would require a $40 \%$ reduction in transmission potential. Thus, targeting sexually mature male hosts alone could reduce endemic TBE cycles and would seem an appropriate strategy in order to maximise efficiency of a TBE control program. By contrast, we examined the scenario where the identity of the transmission cohort was unknown.

The consequence of randomly removing hosts from our system was that the percentage reduction in transmission potential was equivalent to the percentage of the population removed, i.e. 20/20 (Fig. 4). Clearly, this was a less efficient control method than targeting the transmission cohort. The importance of identifying the transmission cohort was additionally apparent when we targeted the 'wrong' hosts. Removing functional groups in order of those least likely to be involved in transmission potential (i.e. sexually mature females, etc. see Fig. 3) we found $20 \%$ of the population would be accountable for just $7 \%$ of the transmission potential (Fig. 4). Thus the $40 \%$ reduction in transmission potential of TBE associated with reducing $R_{0}$ to unity was not achieved until $81 \%$ of the population had been removed. This has important consequences for the control of TBE, but how generally can we apply these findings to other vectorborne diseases and their control? This all depends on whether we can predict which hosts will carry the heavy parasite infestations for a variety of host-parasite systems.

The pattern of male-biased parasitism has been observed in a number of vertebrate taxa, including mammals (e.g. Schalk and Forbes, 1997; Moore and Wilson, 2002) and birds (Poulin, 1996) as well as specifically within tickrodent systems (Randolph, 1977; Ostfeld et al., 1996;
Sorensen and Moses, 1998). In addition to their high susceptibility males tend to have greater exposure to parasites owing to their large home ranges and increased activity. The underlying ultimate, or evolutionary cause of the male sex-biased parasitism in vertebrates has been attributed to the immuno-competence handicap hypothesis, (Folstad and Karter, 1992; Hamilton and Zuk, 1982; Schalk and Forbes, 1997). However, Moore and Wilson (2002) find the sex-bias is attributable to body size (body mass) and where sexual size dimorphism favours females the parasitism will be female biased. They posit the ultimate mechanism behind the sex-bias is therefore resource allocation and not the immuno-suppressive effects of testosterone. The increase in transmission potential we found associated with large body size may be attributed to this resource allocation trade-off, but also a larger body may provide a greater surface area for parasites to target (Arneberg et al., 1998). Additionally behavioural studies have observed larger-sized animals hosting high ectoparasite intensity since 'the smaller they come, the harder they groom' owing to the relative costs of parasitism being far greater for smallersized animals than large ones (Mooring and Hart, 1997; Mooring et al., 2000).

Our empirical observation would seem to support Moore and Wilson's (2002) findings since for all functional groups, regardless of sex we observed an increase in transmission potential associated with increasing body mass (Fig. 3). Additionally males were more likely to support transmission potential than females. An unexpected exception was that sexually immature females had greater transmission potential than sexually immature males (Fig. 3). We attribute this to the greater variance in body mass of sexually immature females than males. Therefore targeted control methods that concentrate on the larger individuals in the population may prove most successful in disproportionately reducing $R_{0}$. We posit the most efficient control method for reduction of TBE prevalence would target the rodent hosts that are sexually mature males of high body mass. Owing to the sex-biased patterns in parasitism observed in a range of vertebrate host-macroparasite systems this recommendation could be extended to other pathogen and parasite control programs where sexual size dimorphism is male biased.

Targeted control of arthropod vectors upon their hosts has economic and ecological advantages over typical vector control methods. Typically, vector control can be directed at the parasite upon the host, or at the parasite within the environment. Heavy and persistent application of insecticide/ acaricide to an entire population may rapidly lead to resistance of the parasite (Hemingway and Ranson, 2000), whilst use of an insecticide in the environment may affect non-target arthropods. Therefore by minimising insecticide/ acaricide application through targeting key hosts we may drastically reduce the long-term economic costs of a control program.

The ecology of the host and the vector cannot be ignored 
when considering the temporal aspect of control. In terms of rodent dynamics we found the transmission cohort is most prominent in the population during the spring months. At this time period the transmission cohort consisted of the previous year's males that have over-wintered and both males and females were sexually mature and at their highest body mass, thus representing cohorts of the greatest and smallest transmission potential. Their role in terms of TBE transmission potential is timely since they are present when a peak in larval and nymphal activity is occurring (Randolph et al., 1996). Removal of the females at this time will result in highly inefficient disease control and may even act to focus the transmission onto the males and therefore increase $R_{0}$. Whereas targeting the sexually mature males at this time period may significantly reduce $R_{0}$. Therefore the most effective time to carry out targeted TBE control would be at this high transmission risk period when the composition of the population in terms of functional groups is most predictable.

Although sexually mature males of high body mass are more likely to be in the transmission cohort we know that individuals do change their body mass and breeding status, and as such it would be difficult to predict the contribution to transmission potential of these individual hosts over time. However, males that are expected to remain sexually mature and, as a consequence, with high body mass are the territorial males. As such we propose these males play an important role within the functional group of sexually mature males, which are potentially the 'key' to TBE virus persistence.

An initial step in epidemiological studies and control of parasites or parasitic infection is quantifying the contributions of hosts to the persistence of the parasite. Here we have identified qualitatively that a relatively small proportion of the host population (20\%) is responsible for the majority of transmission (80\%) and quantitatively identified the epidemiological stratifications within the host population. In terms of disease control, we have identified functional groups that are and are not implicated in transmission potential. In a variety of vertebrate systems the transmission cohort can be expected to consist of sexually mature males. Alternatively where there is a female size bias the pattern of host-parasite associations suggests that large hosts should be targeted. Furthermore if control is carried out at the time of year when these key hosts are dominant and transmission potential is at its highest then disease control may be highly efficient. By implementing targeted control programs we can reduce the cost and effort associated with extensive parasite and pathogen control programs. Failure to include key functional groups in control measures, for example, random targeting of hosts is likely to result in small and inefficient reductions in transmission. (Fig. 4). If we unintentionally initially focus on those hosts that contribute negligibly towards transmission potential then the remaining untargeted population could be highly efficient at transmission and $R_{0}$ may even increase.

\section{Acknowledgements}

This project was in part financed by Centro di Ecologia Alpina of the Autonomous Province of Trento (Italy). We are grateful to Darren Shaw for providing the Splus function nb.fit that allowed us to estimate the parameter $k$. SEP has a NERC funded Studentship with a CEH, Oxford CASE Award.

\section{References}

Anderson, R.M., May, R.M., 1991. Infectious Diseases of Humans: Dynamics and Control. Oxford University Press, Oxford, UK.

Arneberg, P., Skorping, A., Read, A., 1998. Parasite abundance, body size, life histories and the energetic equivalence rule. Am. Nat. 151, 497-513.

Boulinier, T., Ives, A.R., Danchin, E., 1996. Measuring aggregation of parasites at different host population levels. Parasitology 112, 581-587.

Crawley, M.J., 2002. Statistical Computing: an Introduction to Data Analysis using S-Plus. Wiley, New York, NY.

Elston, D.A., Moss, R., Boulinier, T., Arrowsmith, C., Lambin, X., 2001. Analysis of aggregation, a worked example: numbers of ticks on red grouse chicks. Parasitology 122, 563-569.

Folstad, I., Karter, A.J., 1992. Parasites, bright males, and the immunocompetence handicap. Am. Nat. 139, 603-622.

Hamilton, W.D., Zuk, M., 1982. Heritable true fitness and bright birds-a role for parasites. Science 218, 384-387.

Haukisalmi, V., Henttonen, H., 1999. Determinants of helminth aggregation in natural host populations: individual differences or spatial heterogeneity? Ecography 22, 629-636.

Hemingway, J., Ranson, H., 2000. Insecticide resistance in insect vectors of human disease. Annu. Rev. Entomol. 45, 371-391.

Hudson, P.J., Rizzoli, A., Rosà, R., Chemini, C., Jones, L.D., Gould, E.A., 2001. Tick-borne encephalitis virus in northern Italy: molecular analysis, relationships with density and seasonal dynamics of Ixodes ricinus (Acari: Ixodidae). Med. Vet. Entomol. 15, 304-313.

Jones, L.D., Davies, C.R., Steele, G.M., Nuttall, P.A., 1987. A novel mode of arbovirus transmission involving a nonviraemic host. Science 37, $775-777$.

Koch, R., 2000. The 80/20 principle: The secret of achieving more with less. Nicholas Brealey Publishing Ltd, London.

Labuda, M., Jones, L.D., Williams, T., Danielova, V., Nuttall, P.A., 1993. Efficient transmission of tick-borne encephalitis virus between co-feeding ticks. J. Med. Entomol. 30, 295-299.

Labuda, M., Kozuch, O., Zuffova, E., Eleckova, E., Hails, R.S., Nuttall, P.A., 1997. Tick-borne encephalitis virus transmission between ticks co-feeding on specific immune natural rodent hosts. Virology 235, $138-143$

Lorenz, M.O., 1905. Methods for measuring the concentration of wealth. Am. Stat. Assoc. 9, 209-219.

Moore, S.L., Wilson, K., 2002. Parasites as a viability cost of sexual selection in natural populations of mammals. Science 297, 2015-2018.

Mooring, M.S., Hart, B.L., 1997. Self-grooming in impala mothers and lambs: testing the body size and tick challenge principle. Anim. Behav. 53, 925-934.

Mooring, M.S., Benjamin, J.E., Harte, C.R., Herzog, N.B., 2000. Testing the interspecific body size principle in ungulates: the smaller they come, the harder they groom. Anim. Behav. 60, 35-45. 
Ostfeld, R.S., Miller, M.C., Hazler, K.R., 1996. Causes and consequences of tick (Ixodes scapularis) burdens on white-footed mice (Peromyscus leucopus). J. Mammal. 77, 266-273.

Poulin, R., 1993. The disparity between observed and uniform distributions: a new look at parasite aggregation. Int. J. Parasitol. 23 , 937-944.

Poulin, R., 1996. Sexual inequalities in helminth infections: a cost of being a male. Am. Nat. 147, 287-295.

Randolph, S.E., 1977. Patterns of distribution of the tick Ixodes trianguliceps Birula on its hosts. J. Anim. Ecol. 46, 451-474.

Randolph, S.E., 1998. Ticks are not insects: consequences of contrasting vector biology for transmission potential. Parasitol. Today 14, 186-192.

Randolph, S.E., Gern, L., Nuttall, P.A., 1996. Co-feeding ticks: epidemiological significance for tick-borne pathogen transmission. Parasitol. Today 12, 472-479.

Randolph, S.E., Miklisova, D., Lysy, J., Rogers, D.J., Labuda, M., 1999. Incidence from coincidence: patterns of tick infestations on rodents facilitate transmission of tick-borne encephalitis virus. Parasitology $118,177-186$.

Schalk, G., Forbes, M.R., 1997. Male biases in parasitism of mammals: effects of study type, host age, and parasite taxon. Oikos 78, 67-74.
Shaw, D.J., Dobson, A.P., 1995. Patterns of macroparasite abundance and aggregation in wildlife populations. A quantitative review. Parasitology 111 (Suppl), S111-S133.

Shaw, D.J., Grenfell, B.T., Dobson, A.P., 1998. Patterns of macroparasite aggregation in wildlife host populations. Parasitology 117, $597-610$.

Sonenshine, D.E., 1992. Biology of Ticks, vol. 1. Oxford University Press, New York, NY.

Sorensen, T.C., Moses, R.A., 1998. Host preferences and temporal trends of the tick Ixodes angustus in North-central Alberta. J. Parasitol. 84, 902-906.

Wilson, K., Bjornstad, O.N., Dobson, A.P., Merler, S., Poglayen, G., Randolph, S.E., Read, A.F., Skorping, A., 2002. Heterogeneities in macroparasite infections: patterns and processes. In: Hudson, P.J., Rizzoli, A., Grenfell, B.T., Heesterbeek, H., Dobson, A.P. (Eds.), The Ecology of Wildlife Diseases, Oxford University Press, Oxford.

Woolhouse, M.E.J., Dye, C., Etard, J.F., Smith, T., Charlwood, J.D., Garnett, G.P., Hagan, P., Hii, J.L.K., Ndhlovu, P.D., Quinnell, R.J., Watts, C.H., Chandiwana, S.K., Anderson, R.M., 1997. Heterogeneities in the transmission of infectious agents: implications for the design of control programs. Proc. Natl Acad. Sci. USA 94, 338-342. 